\title{
NOROVIRUS INFECTION IN BELARUS: OCCURRENCE AND MOLECULAR EPIDEMIOLOGY
}

\author{
Natallia Uladzimirauna Paklonskaya', Tamara Vasil'evna Amvrosieva'1, Kanstantsin Leanidavich Dziadziulia', \\ Natallia Mikalaeuna Baranouskaya ${ }^{1}$, Elena Petrovna Kishkurno², Nina Leonidovna Kluiko³ \\ ${ }^{1}$ Republican Research and Practical Centre for Epidemiology and Microbiology, Minsk, Belarus \\ 2Belarusian Medical Academy of Post-Graduate Education, Minsk, Belarus \\ ${ }^{3}$ City Children's Clinical Hospital of Infectious Diseases, Minsk, Belarus
}

\section{SUMMARY}

The objective of the study is to analyze molecular epidemiologic surveillance for norovirus infection in Belarus over the past five years (2009-2013). Laboratory diagnostics was carried out by RT-PCR in 684 patients. Two regions of norovirus genome, localized in RNA-polymerase and capsid protein genes, were used for phylogenetic analysis.

Noroviruses were predominant causative agents in adults and second only to rotaviruses in children, they also prevailed among aetiological agents of outbreaks ( $66.7 \%$ of outbreaks). In 2009-2013, the major norovirus genotype was GII.4 (58.3\% of all genotyped isolates). Genovariant GII.4 2006b circulated in 2009 and 2010, genovariant GII.4 2009 New Orleans - in 2010 and 2012. In addition to Gll.4, genotypes Gll.6 (16.6\%), GII.2 (4.1\%), GII.3 (2.2\%), and recombinant genotypes GII.g-GIl.12 and GII.g-GIl.1 (10.4\% and 8.3\%, respectively) circulated in Belarus.

The findings indicate a significant contribution of noroviruses in development of sporadic morbidity and outbreaks of acute gastroenteritis in Belarus. Outbreaks or prominent increases of sporadic morbidity were mostly due to the emergence of a new genotype, or an epidemic genovariant.

Key words: norovirus, acute gastroenteritis, group and sporadic morbidity, genotype

Address for correspondence: N. Paklonskaya, Republican Research and Practical Centre for Epidemiology and Microbiology, 23, Philimonov str., 220114, Minsk, Republic of Belarus. E-mail: labsanvir@gmail.com

\section{INTRODUCTION}

Norovirus (NoV) is a genus of small non-enveloped viruses with (+)ssRNA genome in the Caliciviridae family. The genus comprises 5 distinct virus genogroups, 3 of which (I, II, and IV) are human pathogens (1). In the mid 1990's, a significant increase of NoV infection incidence was registered worldwide; the reasons for such elevation in virus activity are not fully understood. At the present time, noroviruses are the most common cause of acute gastroenteritis in adults and dominant aetiological agents of acute enteric infections outbreaks, including foodborne infections (2, 3 ). In paediatric patients noroviruses are also common cause of acute gastroenteritis second only to rotaviruses (4).

Noroviruses are characterized by extremely high level of genetic variability underlying their genetic and phenotypic diversity (5). The latter factor accounts for rapid emergence and concurrent circulation of multiple antigenic variants, which, on the one hand, prevents formation of sustainable immune response in the course of natural immunization, and, on the other hand, hinders development of effective vaccines (6). Due to lack of specific means for treatment and prevention of NoV infection, the most productive current strategy to restrain transmission of the disease involves regular epidemiological surveillance for the circulation of viral agents, based on the results of laboratory control procedures and molecular-epidemiological investigations. Such surveillance permits to detect in a timely manner the emergence and dissemination of the new epidemic genovariants capable of eliciting significant rise of morbidity and to take opportune anti-epidemic measures.
In Belarus, routine laboratory diagnostics and research into molecular epidemiology of NoV infection, including genotyping of its agents, have been under way since 2009. Concurrently, investigations of aetiologic structure of acute enteric infection and gastroenteritis outbreaks, caused by adeno-, astro-, and sapoviruses, have been carried out.

The present paper summarizes data collected in Belarus over the past 5 years (2009-2013) in the scope of molecular-epidemiological surveillance for NoV infection agents.

\section{MATERIALS AND METHODS}

Stool samples were collected from 684 patients, including 577 children (397 children younger than 5 years, 180 children aged 5-18 years) and 107 adults within 24-48 hours of onset of the disease. All patients were hospitalized during 2009-2013 with diagnosis of acute gastroenteritis $(n=496)$, acute enteric infection $(n=88)$, food toxicoinfection $(n=31)$, acute enteritis $(n=44)$, acute enterocolitis $(n=17)$, acute gastritis $(n=4)$, and hemocolitis $(n=4)$.

Commercial kits RIBO-SORB and RIBOPREP (AmpliSens, Russia) were used to isolate RNA from clinical samples in accordance with manufacturer's directions. Reverse transcription was carried out with RevertAid kit (Fermentas, Lithuania), or REVERTA kit (AmpliSens, Russia).

Differential laboratory diagnostics was performed with the help of commercial kits Rotavirus/Norovirus/Astrovirus-FL, AEI screen-FL, and Norovirus 1,2 genotypes-EPh (AmpliSens ${ }^{\circledR}$, Russia), as well as with multiple primer sets described elsewhere (7-9). 
Two fragments of norovirus genome were selected for sequencing: a $340 \mathrm{nt}$ fragment in 3' RdRp region, and a $280 \mathrm{nt}$ portion of VP1 gene $(7,8)$. DNA sequencing was performed by chain termination in thermocyclic reaction with the help of DTCS Quick Start Kit and automatic DNA-analyzer CEQ8000 (Beckman Coulter).

BLAST (Basic Local Alignment Search Tool) was used for homologous sequences search in the NCBI database (10). Computer sequence analysis (multiple alignment, calculation of evolutionary distances, phylogenetic reconstruction, and testing of tree topology) was performed with MEGA (Molecular Evolutionary Genetics Analysis) software package, version 6.0 (11). Maximum likelihood method and corrected Akaike information criterion (AICc) were employed to determine optimal mathematic model of nucleotide substitution. Phylogenetic reconstruction was based on neighbour joining (NJ) and maximum likelihood (ML) algorithms implemented in MEGA 6.0. Bootstrap analysis was used for testing of the phylogenetic trees topology with 1000 pseudoreplicates analyzed for each tree (12).

\section{RESULTS}

Results of laboratory diagnostics performed in the setting of sporadic morbidity for selected patients with clinical diagnosis of acute enteric infection in the years of 2009-2013 as well as data on the aetiology of outbreaks suggest considerable share of noroviruses in the structure of viral enteric infections. In general, noroviruses were second only to rotaviruses in terms of detection rate (Fig. 1). They were predominant causative agents of

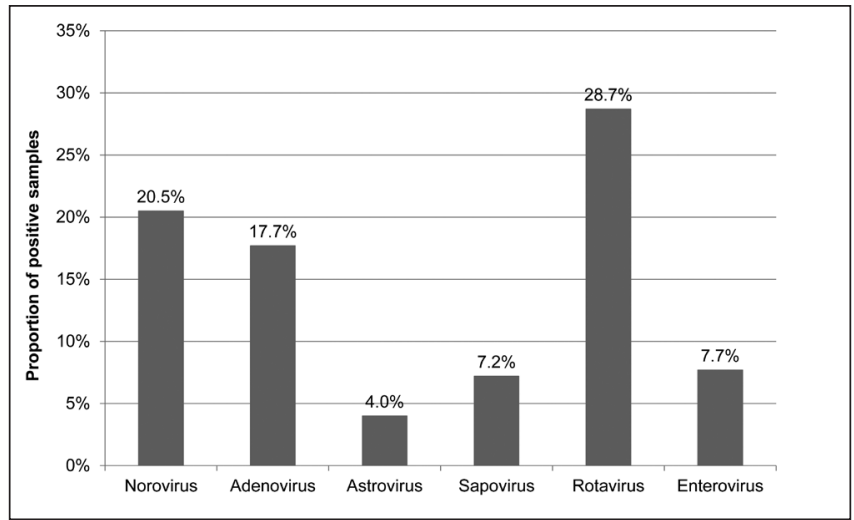

Fig. 1. NoV share in the aetiology of viral acute enteric infections.

acute enteric infections in adults: $30.0 \%$ of acute gastroenteritis cases were caused by norovirus, whereas only $8.6 \%$ by rotavirus. Genogroup II NoV were prevalent aetiologic factors of intestinal disease group morbidity and caused $60.0 \%$ of outbreaks, whereas Sapovirus only $13.3 \%$, Salmonella spp. $6.7 \%$, Rotavirus $6.7 \%$, Norovirus I 6.7\%, and Enterovirus 6,7\% of registered episodes. Detection rate of NoV from genogroup II had clear seasonal variation: proportion of sporadic morbidity associated with NoV GII was 3 times higher in winter than in summer $(29.6 \%$ vs $10.6 \%$, respectively). However, occurrence of NoV infection group morbidity was almost on the same level all year round.

From 2009-2013, a total of 15 group morbidity episodes registered in different seasons were investigated to establish the underlying aetiology (Table 1). One hundred and four patients

Table 1. General characteristics of acute enteric infections outbreaks in Belarus, 2009-2014

\begin{tabular}{|c|c|c|c|c|c|c|}
\hline $\begin{array}{l}\text { Data } \\
\text { (month year) }\end{array}$ & Region & Clinical diagnosis & $\begin{array}{l}\text { Number of as- } \\
\text { sessed patients }\end{array}$ & Age & Detected agent(s) & $\begin{array}{c}\text { Number of patients } \\
\text { with established } \\
\text { aetiology }\end{array}$ \\
\hline May 2009 & Minsk & AGE & 8 & $18-20$ & Sapovirus & 6 \\
\hline \multirow{3}{*}{ February 2010} & \multirow{3}{*}{ Minsk region } & \multirow{3}{*}{ AGE } & \multirow{3}{*}{10} & \multirow{3}{*}{$18-20$} & Sapovirus & 6 \\
\hline & & & & & Norovirus II & 1 \\
\hline & & & & & Enterovirus & 3 \\
\hline March 2010 & Minsk & AGE & 8 & $45-86$ & Norovirus II & 7 \\
\hline May 2010 & Minsk & AGE & 15 & $16-17$ & Norovirus II & 13 \\
\hline \multirow{2}{*}{ February 2011} & \multirow{2}{*}{ Minsk } & \multirow{2}{*}{ AGE } & \multirow{2}{*}{5} & \multirow{2}{*}{$18-25$} & Norovirus II & 4 \\
\hline & & & & & Enterovirus & 2 \\
\hline January 2012 & Lyahovichi & AGE & 11 & $11-18$ & Norovirus II & 9 \\
\hline May 2012 & Luninets & FTI & 5 & $7-8$ & Norovirus II & 5 \\
\hline July 2012 & Borisov & AGE & 6 & $7-12$ & Enterovirus & 6 \\
\hline July 2012 & Myadel & AGE & 5 & $9-15$ & Norovirus I & 4 \\
\hline July 2012 & Minsk & AGE & 6 & $18-20$ & Norovirus II & 2 \\
\hline October 2012 & Luninets & AGE & 3 & $16-17$ & Norovirus II & 2 \\
\hline \multirow{2}{*}{ July 2013} & \multirow{2}{*}{ Minsk region } & \multirow{2}{*}{ AGE } & \multirow{2}{*}{7} & \multirow{2}{*}{$6-13$} & Norovirus II & 6 \\
\hline & & & & & Enterovirus & 1 \\
\hline August 2013 & Minsk region & AGE & 6 & $18-20$ & Salmonella spp. & 5 \\
\hline October 2013 & Minsk region & AGE & 6 & $18-20$ & Rotavirus A & 5 \\
\hline January 2014 & Minsk region & AGE & 2 & $10-12$ & Norovirus II & 2 \\
\hline
\end{tabular}


were assessed in regard of viral (Rotavirus, Norovirus GI and GII, Astrovirus, Adenovirus, Sapovirus, and Enterovirus), or bacterial (Salmonella, Shigella and Campylobacter) intestinal infections. Almost all of the reported episodes occurred in closed or semi-closed communities, such as children's summer camps, boarding schools, military bases, etc. Age of the patients ranged from 6 to 86 years.

Primary clinical diagnosis was acute gastroenteritis. The majority of group morbidity episodes were caused by a single infectious agent $(73.3 \%$, positive $11 /$ total 15$)$, while 4 of them were associated with concomitant transmission of several (2-3) viruses. In such cases the pathogen revealed in more than one third of examined patients was considered the aetiologic agent of the outbreak. The data indicate dominant role of NoV as the cause of acute enteric infections group morbidity: $66.7 \%$ of episodes were caused by NoV from genogroups I and II, comprising 60\% of members of genogroup II and $6.7 \%$ of members of genogroup I. In half of the reported episodes noroviruses were the only aetiologic agents identified.

Genotyping of NoV isolates and molecular-epidemiological surveillance for NoV infection were based on sequence analysis of two virus genome fragments. In total, $48 \mathrm{NoV}$ isolates from patients of different age were assessed. The viruses were collected during periods of sporadic morbidity and at the time of outbreaks. Two genomic regions were selected for molecularepidemiological investigations: a fragment of major capsid protein VP1 gene and a fragment of RNA-dependent RNA-polymerase gene, which allow molecular typing and identification of known

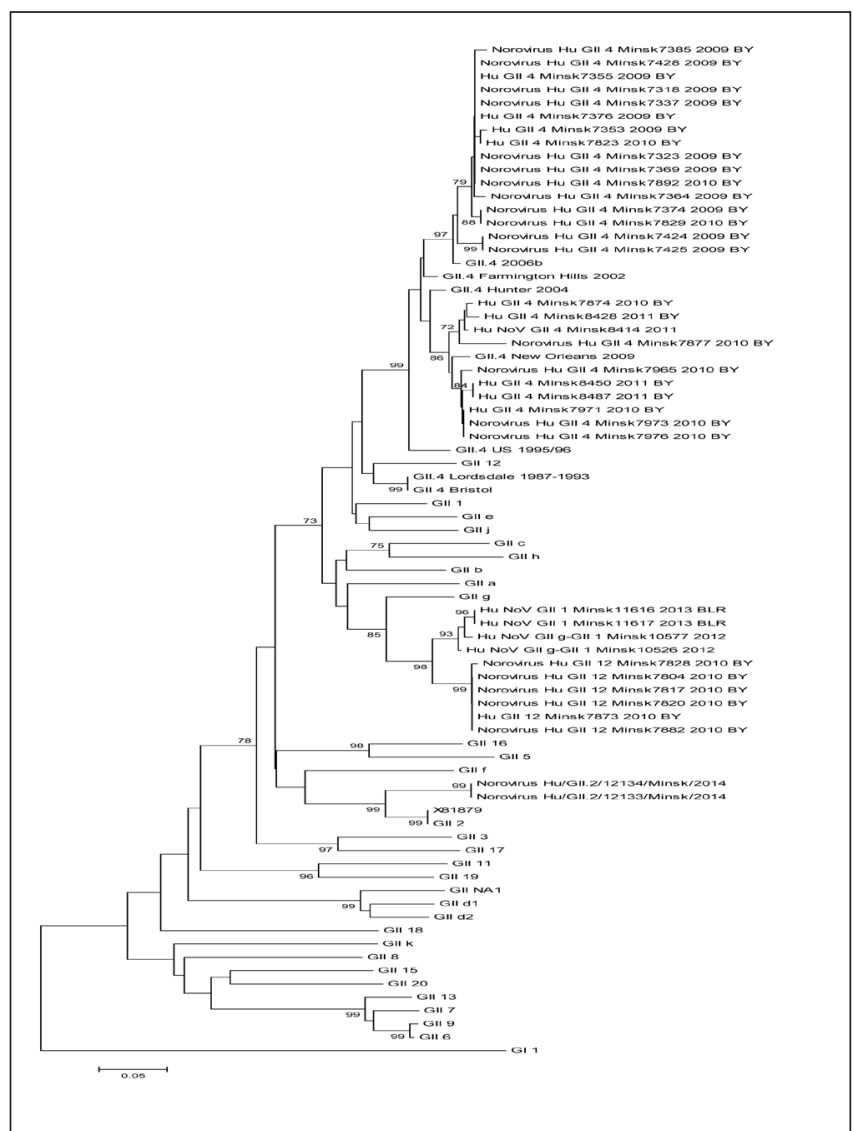

Fig. 2. NJ phylogenetic tree based on nucleotide sequence analysis of RNA-polymerase gene fragment of $38 \mathrm{NoV}$ isolates from patients with acute enteric infection (Belarus, 2009-2014). epidemic genovariants of NoV. For 16 virus isolates analysis was performed in parallel on 2 genomic regions, this provided opportunity to identify recombinant genovariants. Analysis of 38 isolates was based on RNA-polymerase gene fragment only, and for 25 isolates a portion of VP1 gene was used. Neighbour joining and maximum likelihood phylogenetic trees derived from sequence analysis of the two genomic regions are presented in Figures 2 and 3.

The results showed that $58.3 \%$ of NoV isolates $(28 / 48)$ belonged to genotype GII.4, currently the most prevalent NoV genotype worldwide. Within genotype GII.4 two global genovariants were identified: $33.3 \%$ of NoV isolates $(16 / 48)$ belonged to genovariant GII.4 2006b, whereas $25.0 \%$ of NoV isolates (12/48) were identified as members of genovariant GII.4 2009 New Orleans. In addition to the major genotype GII.4, other NoV genotypes circulated in Belarus: GII.6 (16.6\%), recombinant genotypes GII.g-GII.12 and GII.g-GII.1 (10.4\% and $8.3 \%$, respectively), GII.2 (4.1\%), and GII.3 (2.2\%).

\section{DISCUSSION}

Analysis of the presented NoV phylogenetic reconstruction allows us to describe molecular epidemiology of NoV in Belarus between 2009 and 2013. As can be expected, the majority of genotyped NoV isolates belonged to the most prevalent GII.4 NoV genotype. To date, within this genotype 6 distinct genovariants with global circulation have been identified, which accounted for

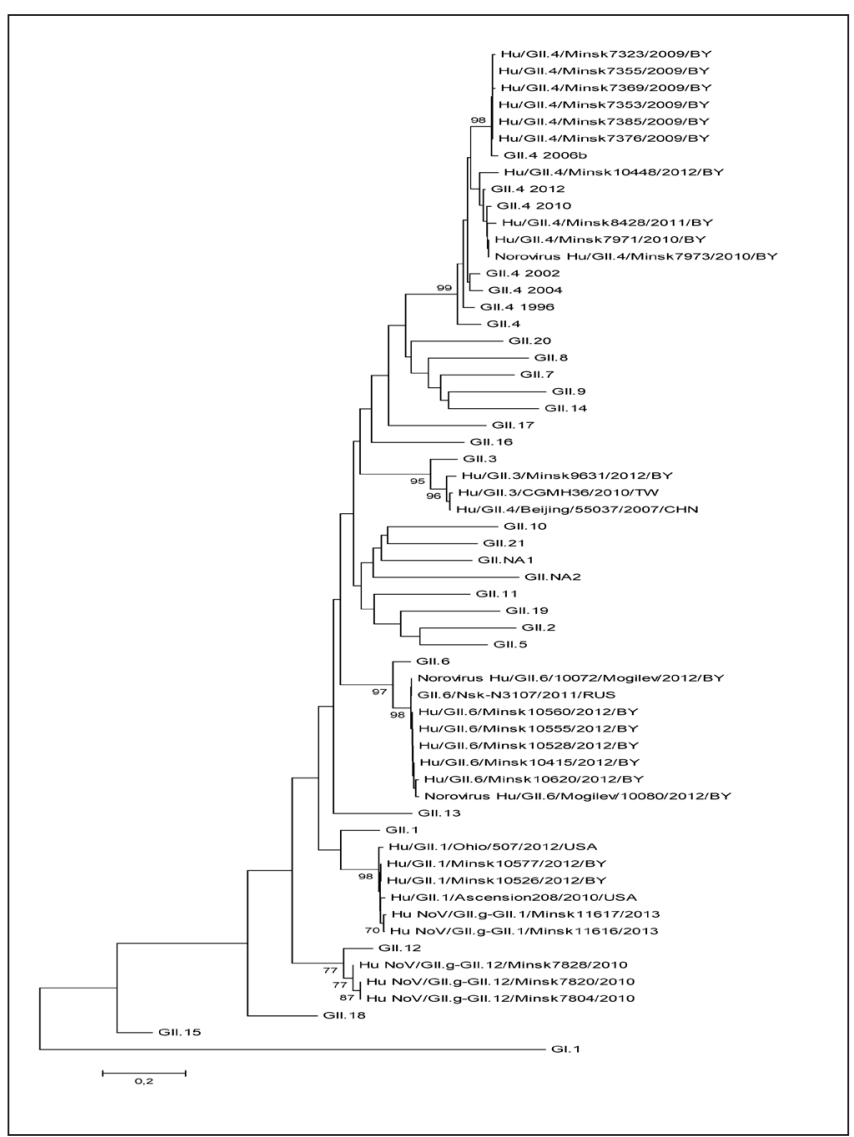

Fig. 3. ML phylogenetic tree based on nucleotide sequence analysis of 280 nt VP1 gene fragment of $25 \mathrm{NoV}$ isolates from patients with acute enteric infection (Belarus, 2009-2013). 
$62-80 \%$ of NoV infection outbreaks in different countries (13, 14). These genovariants include US $1995 / 96$ (1996), Farmington Hills (2002), Hunter (2004), 2006b (2007-2008), New Orleans (2009-2012), and Sydney (2012-2014) strains. Phylogenetic analysis of Belarusian NoV isolates revealed local circulation of 2 out of 6 global genovariants belonging to genotype GII.4: sporadic morbidity of 2009-2010 was caused by genovariant GII.4 2006b, while the causative agent of 3 episodes of group morbidity (March and May 2010, and January 2011) was identified as a member of genovariant GII.4 2009 New Orleans. In 2011-2012, circulation of the latter genovariant continued and NoV GII.4 2009 New Orleans isolates were detected in patients with sporadic cases of NoV infection.

Genotype GII.3 was represented by a single agent that caused an episode of group morbidity during winter 2012; that was the only instance when circulation of this strain was registered in the country. Similarly, genotype GII.2 caused a single episode of group morbidity in children in 2014, but was never detected in the setting of sporadic morbidity. Genotype GII.6 was the culprit in two group morbidity episodes - in July and October 2012 - and was also identified in sporadic cases of NoV infection. Genotype GII.g-GII.12 for the first time emerged in Belarus in 2010 together with epidemic genovariant GII.4 New Orleans caused significant increase of sporadic NoV infection incidence during winter 2011 (the proportion of $\mathrm{NoV}$-associated acute enteric infections was 1.9-1.4 times higher in winter 2011 than in 2009-2010, data not showed). Recombinant genotype GII.g-GII.1 was registered in sporadic cases of NoV infection in summer 2012 and later, in July 2013, caused an episode of group morbidity in children.

Of note, outbreaks and seasonal increases of morbidity caused by recombinant NoV genotypes GII.g-GII.12 and GII.g-GII.1 were almost simultaneously observed in different countries worldwide. Thus, in 2010, genotype GII.g-GII.12 was the causative agent of winter increase of morbidity in USA ( $16 \%$ of all reported outbreaks) (15), while genotype GII.g-GII.1 was prevalent in Belgium and caused a major foodborne infection outbreak in a German hospital in 2012 (16).

\section{CONCLUSION}

In summary, it is evident that over the past 5 years significant level of genetic diversity and rapid succession of circulating NoV genotypes and genovariants were observed in Belarus. The new epidemic genovariants emerged almost at the same time, these agents spread in other parts of the world and generally were accompanied by increases of morbidity, group or sporadic. These findings support the necessity for regular molecular-epidemiological surveillance for NoV infection which will allow early detection of new virus genovariants and prognostic assessment of their epidemic potential.

\section{REFERENCES}

1. Zheng DP, Ando T, Fankhauser RL, Beard RS, Glass RI, Monroe SS. Norovirus classification and proposed strain nomenclature. Virology. 2006 Mar 15;346(2):312-23.

2. Lopman BA, Reacher MH, Van Duijnhoven Y, Hanon FX, Brown D, Koopmans M. Viral gastroenteritis outbreaks in Europe, 1995-2000. Emerg Infect Dis. 2003 Jan;9(1):90-6.

3. Widdowson MA, Monroe SS, Glass RI. Are noroviruses emerging? Emerg Infect Dis. 2005 May;11(5):735-7.

4. Patel MM, Widdowson MA, Glass RI, Akazawa K, Vinjé J, Parashar UD. Systematic literature review of role of noroviruses in sporadic gastroenteritis. Emerg Infect Dis. 2008 Aug;14(8):1224-31.

5. Victoria M, Miagostovich MP, Ferreira MS, Vieira CB, Fioretti JM, Leite JP, et al. Bayesian coalescent inference reveals high evolutionary rates and expansion of Norovirus populations. Infect Genet Evol. 2009 Sep;9(5):927-32.

6. Lindesmith LC, Donaldson EF, Baric RS. Norovirus GII.4 strain antigenic variation. J Virol. 2011 Jan;85(1):231-42.

7. Vinjé J, Vennema H, Maunula L, von Bonsdorff CH, Hoehne M, Schreier $\mathrm{E}$, et al. International collaborative study to compare reverse transcriptase PCR assays for detection and genotyping of noroviruses. J Clin Microbiol. 2003 Apr;41(4):1423-33.

8. Kojima S, Kageyama T, Fukushi S, Hoshino FB, Shinohara M, Uchida $\mathrm{K}$, et al. Genogroup-specific PCR primers for detection of Norwalk-like viruses. J Virol Methods. 2002 Feb;100(1-2):107-14.

9. Yan H, Yagyu F, Okitsu S, Nishio O, Ushijima H. Detection of norovirus (GI, GII), Sapovirus and astrovirus in fecal samples using reverse transcription single-round multiplex PCR. J Virol Methods. 2003 Dec;114(1):37-44.

10. Altschul SF, Gish W, Miller W, Myers EW, Lipman DJ. Basic local alignment search tool. J Mol Biol. 1990 Oct 5;215(3):403-10.

11. Tamura K, Stecher G, Peterson D, Filipski A, Kumar S. MEGA6: Molecular Evolutionary Genetics Analysis version 6.0. Mol Biol Evol. 2013 Dec;30(12):2725-9.

12. Felsenstein J. Confidence limits on phylogenies: an approach using the bootstrap. Evolution. 1985;39(4):783-91.

13. Bull RA, White PA. Mechanisms of GII.4 norovirus evolution. Trends Microbiol. 2011 May;19(5):233-40.

14. Eden JS, Hewitt J, Lim KL, Boni MF, Merif J, Greening G, et al. The emergence and evolution of the novel epidemic norovirus GII.4 variant Sydney 2012. Virology. 2014 Feb;450-451:106-13.

15. Vega E, Vinjé J. Novel GII.12 norovirus strain, United States, 2009-2010. Emerg Infect Dis. 2011 Aug;17(8):1516-8.

16. Hoffmann D, Mauroy A, Seebach J, Simon V, Wantia N, Protzer U. New norovirus classified as a recombinant GII.g/GII.1 causes an extended foodborne outbreak at a university hospital in Munich. J Clin Virol. 2013 Sep;58(1):24-30. 